\title{
Targeted intraarterial anti-VEGF therapy for medically refractory radiation necrosis in the brain
}

\author{
Shervin R. Dashti, MD, PhD, ${ }^{1}$ Aaron Spalding, MD, PhD, ${ }^{2}$ Rob J. Kadner, MD, ${ }^{1}$ Tom Yao, MD, ${ }^{1}$ \\ Arooshi Kumar, BS, ${ }^{3}$ David A. Sun, MD, PhD, ${ }^{1,2}$ and Renato LaRocca, MD ${ }^{2}$ \\ ${ }^{1}$ Norton Neuroscience Institute and ${ }^{2}$ Norton Cancer Institute, Norton Healthcare; and ${ }^{3}$ University of Louisville School of
Medicine, Louisville, Kentucky
}

\begin{abstract}
Radiation necrosis $(R N)$ is a serious complication that can occur in up to $10 \%$ of brain radiotherapy cases, with the incidence dependent on both dose and brain location. Available medical treatment for RN includes steroids, vitamin $\mathrm{E}$, pentoxifylline, and hyperbaric oxygen. In a significant number of patients, however, $\mathrm{RN}$ is medically refractory and the patients experience progressive neurological decline, disabling headaches, and decreased quality of life.

Vascular endothelial growth factor (VEGF) is a known mediator of cerebral edema in RN. Recent reports have shown successful treatment of RN with intravenous bevacizumab, a monoclonal antibody for VEGF. Bevacizumab, however, is associated with significant systemic complications including sinus thrombosis, pulmonary embolus, gastrointestinal tract perforation, wound dehiscence, and severe hypertension. Using lower drug doses may decrease systemic exposure and reduce complication rates. By using an intraarterial route for drug administration following blood-brain barrier disruption (BBBD), the authors aim to lower the bevacizumab dose while increasing target delivery.

In the present report, the authors present the cases of 2 pediatric patients with cerebral arteriovenous malformations, who presented with medically intractable $\mathrm{RN}$ following stereotactic radiosurgery. They received a single intraarterial infusion of $2.5 \mathrm{mg} / \mathrm{kg}$ bevacizumab after hyperosmotic BBBD.

At mean follow-up duration of 8.5 months, the patients had significant and durable clinical and radiographic response. Both patients experienced resolution of their previously intractable headaches and reversal of cushingoid features as they were successfully weaned off steroids. One of the patients regained significant motor strength. There was an associated greater than $70 \%$ reduction in cerebral edema.

Intraarterial administration of a single low dose of bevacizumab after BBBD was safe and resulted in durable clinical and radiographic improvements at concentrations well below those required for the typical systemic intravenous route. Advantages over the intravenous route may include higher concentration of drug delivery to the affected brain, decreased systemic toxicity, and a significantly lower cost.
\end{abstract}

http://thejns.org/doi/abs/10.3171/2014.9.PEDS14198

KEY WORDS bevacizumab; radiation necrosis; radiation adverse effect; blood-brain barrier disruption; arteriovenous malformation; stereotactic radiosurgery; intraarterial chemotherapy; oncology

$\mathrm{R}$ ADIATION necrosis (RN) is a serious complication that can occur in up to $10 \%$ of brain radiotherapy cases, with the incidence dependent on both dose and brain location. Available medical treatment for RN includes steroids, vitamin E, pentoxifylline, and hyperbaric oxygen. ${ }^{9}$ In a significant number of patients, however, RN is medically refractory, and the patients experience progressive neurological decline, disabling headaches, and decreased quality of life.
Vascular endothelial growth factor (VEGF) is a known mediator of cerebral edema in RN. Recent reports have shown successful treatment of $\mathrm{RN}$ with intravenous bevacizumab, a monoclonal antibody for VEGF. Bevacizumab, however, is associated with significant systemic complications including venous thrombosis, pulmonary embolus, gastrointestinal tract perforation, wound dehiscence, and severe hypertension. Using lower drug doses may reduce systemic exposure and decrease complication rates, pro-

ABBREVIATIONS AVM = arteriovenous malformation; BBB = blood-brain barrier; $\mathrm{BBBD}=\mathrm{BBB}$ disruption; $\mathrm{SRS}=$ stereotactic radiosurgery; $\mathrm{RN}=$ radiation necrosis; VEGF = vascular endothelial growth factor.

SUBMITTED April 9, 2014. ACCEPTED September 29, 2014.

INCLUDE WHEN CITING Published online October 31, 2014; DOI: 10.3171/2014.9.PEDS14198.

DISCLOSURE The authors report no conflict of interest concerning the materials or methods used in this study or the findings specified in this paper. 
viding a rationale for further study. By using an intraarterial route for drug administration following blood-brain barrier (BBB) disruption (BBBD), we aimed to lower the bevacizumab dose while increasing target delivery.

\section{Case Reports}

This case report was reviewed by the University of Louisville institutional review board.

\section{Technique for Intraarterial Bevacizumab Infusion After BBB Breakdown}

The patients were premedicated with $6 \mathrm{mg}$ dexamethasone and $1000 \mathrm{mg}$ Keppra. The femoral artery was accessed using the Seldinger technique. A 5-Fr diagnostic catheter was used to catheterize the cervical internal carotid artery ipsilateral to the lesion. Baseline internal carotid artery angiography was performed.

Next, $5 \mathrm{mg}$ Valium and $0.2 \mathrm{mg}$ atropine were administered intravenously. Warm $\left(37^{\circ} \mathrm{C}\right) 25 \%$ mannitol was infused into the ipsilateral cervical carotid artery at a rate of $4 \mathrm{ml} / \mathrm{sec}$ for $30 \mathrm{sec}$. This was followed by infusion of $2.5 \mathrm{mg} / \mathrm{kg}$ bevacizumab in a volume of $100 \mathrm{ml}$ over 14 minutes.

The patients were then monitored in the recovery unit with frequent neurological exams and continuous heart rate and pulse-oximetry measurement for 2 hours. They were then observed overnight in a pediatric intensive care unit, with neurological status and vital signs checked every 2 hours, as well as continuous heart rate and pulse-oximetry monitoring. Both patients were discharged to home on postoperative Day 1. No adverse events were noted.

\section{MRI Signal Quantification}

The MRI scans were retrospectively reviewed by a board-certified neuroradiologist, and the volume of altered FLAIR signal and Gd enhancement was analyzed. The images were viewed on an Aquarius workstation (TeraRecon). Utilizing the segmentation, analysis, and tracking module, the regions of interest were outlined and volumetric data were calculated. The follow-up data were compared with the baseline data to produce a calculation of percentage change from baseline.

\section{Case 1}

History and Examination

A 12-year-old right-handed girl presented with severe headaches. Brain MRI showed a 3.2-cm left posterior frontal arteriovenous malformation (AVM; Spetzler-Martin Grade III) and mild surrounding vasogenic edema. There were no signs of hemorrhage. The patient was initially treated with a 2-week course of steroids and her headaches significantly improved and MRI revealed decreased edema. After considering treatment options including observation, endovascular embolization, and/or resection, the patient and her family chose stereotactic radiosurgery (SRS). She underwent SRS in which a 21-Gy radiation dose was prescribed to the AVM (lesion volume $9.9 \mathrm{~cm}^{3}$ ).

Eight months later, the patient presented with severe headaches and focal seizures affecting her right arm and leg. Brain MRI at this time demonstrated increasing T2 FLAIR signal and new Gd signal on the T1-weighted sequences (Fig. 1 left and right, respectively). The patient was treated with antiepileptic medication for her seizures and oral steroids for her presumed RN. Her seizure episodes resolved, but her severe headache persisted. She was initially started on $24 \mathrm{mg}$ per day of dexamethasone that was tapered over 3 weeks. Because of worsening headache and progressively worsening right hemiparesis, repeated attempts to reduce dexamethasone dose below $6 \mathrm{mg}$ daily proved unsuccessful over the ensuing 9 months. Courses of pentoxifylline, vitamin E, and hyperbaric oxygen were all administered per study protocols, without success. ${ }^{15,24}$ Despite these interventions, her proximal right upper and lower extremities declined to Grade 4/5 strength and her right hand and foot declined to Grade 0/5 strength. She experienced a 60-pound weight gain and severe cushingoid features. She complained of constant severe headache $(10 / 10$ on the visual analog scale). She also experienced severe emotional lability, causing her to withdraw from school.

\section{Intervention}

Given the progressive neurological decline and the lack of alternative therapies, the option of intravenous administration of bevacizumab was initially explored. However, this proved to be not available for our pediatric patient population. Hence, we considered the option of low-dose intraarterial bevacizumab treatment. Our rationale for this approach was to use a smaller dose of bevacizumab in a more directed fashion (intraarterial administration after disrupting the $\mathrm{BBB}$ ), to maintain efficacy while reducing systemic toxicity. ${ }^{14}$ The off-label uses of the medication, as well as the risks, including intracranial hemorrhage, were reviewed in detail. Informed written consent was provided by the parents, and the patient gave her assent. We performed intraarterial infusion of $2.5 \mathrm{mg} / \mathrm{kg}$ bevacizumab after hyperosmotic BBB breakdown. There were no acute complications from the procedure.

\section{Postintervention Course}

Within 12 hours of intraarterial bevacizumab administration, the patient experienced complete resolution of her previously intractable headaches (now a 0 on the 10-point scale). She also felt subjectively stronger on her right side. She was started on a long steroid taper under supervision of the Endocrine Service.

Two months later, brain MRI revealed an $82 \%$ decrease in FLAIR signal and a 6\% decrease in contrast enhancement (Fig. 1 left and right, respectively). There was an associated markedly reduced mass effect. MRI of the brain at 5 months revealed $78 \%$ decreased FLAIR signal and $22 \%$ decreased contrast enhancement (Fig. 1 left and right, respectively).

At 9 months, the patient exhibited a progressive and prominent improvement in her proximal right arm and leg strength. She was able to walk long distances with the help of an electronic drop-foot unit. She has continued to lose weight (13 pounds so far). She experiences occasional moderate headaches (5-6 on a 10-point scale) that would last several hours, but the headaches are not the constant, 

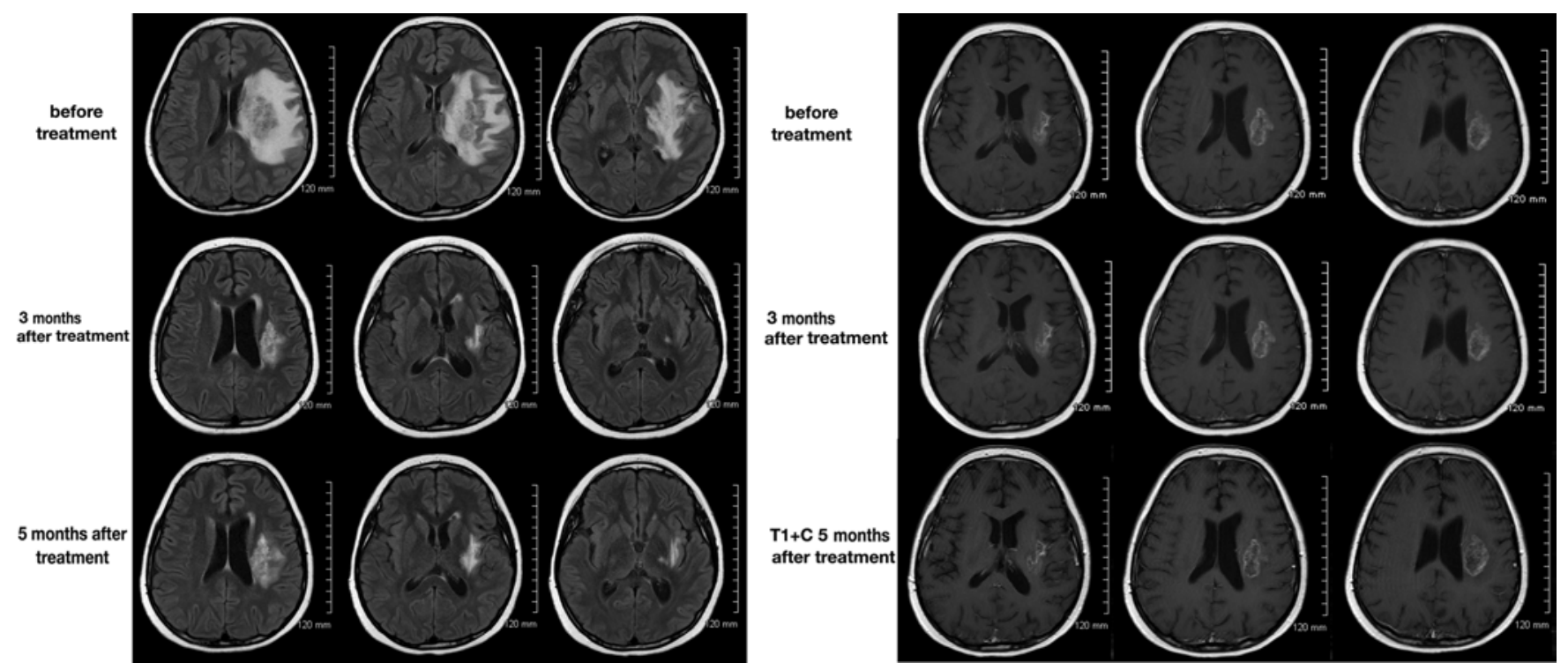

FIG. 1. Case 1. Left: Brain FLAIR MR images showing prominently increased FLAIR signal in the left hemisphere before treatment (upper images). There is a marked $82 \%$ and $78 \%$ decrease in FLAIR signal volumes at 3 months (center images) and 5 months (lower images), respectively, after intraarterial bevacizumab infusion. Right: Brain T1-weighted contrast-enhanced MR images demonstrating signal enhancement before treatment (upper images) and a $6 \%$ and $22 \%$ decrease in contrast enhancement at 3 months (center images) and 5 months (lower images), respectively, after intraarterial bevacizumab infusion.

very severe ones she was experiencing before treatment. Her emotional lability resolved and she was able to go back to school.

\section{Case 2}

History and Examination

An 11-year-old right-handed girl presented with right hemiparesis, headache, nausea, and vomiting. Head CT scanning revealed intraparenchymal and intraventricular hemorrhage and hydrocephalus. She required temporary external ventricular drainage. MRI imaging demonstrated a Spetzler-Martin Grade II AVM of the corpus callosum. She underwent embolization 2 months later with only partial obliteration. She then underwent SRS for the residual AVM nidus with an 18-Gy radiation dose prescribed to a volume of $4.3 \mathrm{~cm}^{3}$.

Within the 1st month after treatment, the patient developed moderate headache, which responded to a 2-week course of steroids. Six months after SRS, she developed mild to moderate headaches, which initially responded to ibuprofen. MRI showed evidence of mild vasogenic edema surrounding the AVM site. Eight months after radiosurgery, she developed intractable headaches that were associated with nausea and vomiting. Repeat MRI demonstrated worsening cerebral edema with new contrast enhancement consistent with $\mathrm{RN}$ in the left frontal lobe (Fig. 2). There were no associated motor or sensory symptoms. A 21-day course of steroid treatment, starting with $24 \mathrm{mg}$ per day of dexamethasone, was initiated. This steroid treatment could not, however, be tapered below $8 \mathrm{mg}$ daily over the ensuing 3 months because of the patient's recurrent severe headache, nausea, and vomiting. Courses of pentoxifylline and vitamin $\mathrm{E}$ were tried without success. Over time, she developed significant steroid-related symptoms. She also required a hospitalization for fluid overload. In less than 3 months, the patient gained over 30 pounds and had a body mass index of 25.5. As a result of her symptoms, she too had to withdraw from school.

\section{Intervention}

Based on the initial experience with Case 1, we discussed the option of intraarterial bevacizumab treatment. The off-label uses of the medication and the risks, including intracranial hemorrhage, were reviewed in detail. An informed written consent form was signed by the parents, and the patient provided assent. The patient underwent intraarterial infusion of $2.5 \mathrm{mg} / \mathrm{kg}$ bevacizumab into the left internal carotid artery after hyperosmotic BBB breakdown. There were no complications.

\section{Postintervention Course}

Immediately after intraarterial bevacizumab administration, the patient experienced complete resolution of her intractable headache, and within 4 weeks she was successfully weaned off steroids. Brain MRI 3 months later revealed a $74 \%$ decrease in FLAIR signal volume and a $33.6 \%$ decrease in contrast enhancement (Fig. 2 left and right, respectively). At 8-month clinical follow-up examination, the patient was free of headaches and was back in school.

\section{Discussion}

Stereotactic radiosurgery has become integral in treatment of brain tumors and AVMs. In up to $10 \%$ of cases, this can lead to RN with significant surrounding vasogenic edema and mass effect. Medical treatment for RN includes steroids, vitamin E, pentoxifylline, and hyperbaric oxygen. Up to $20 \%$ of cases are medically refractory and the patients experience progressive neurological decline and disabling headaches. 

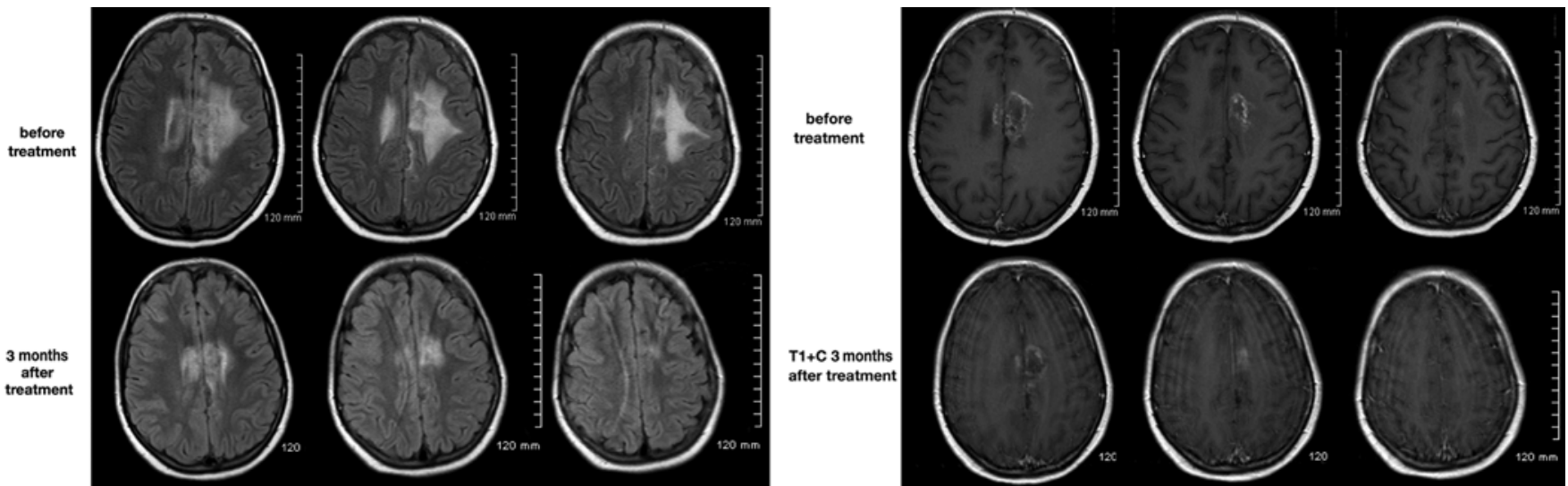

FIG. 2. Case 2. Left: Brain FLAIR MR images showing prominently increased FLAIR signal in the left centrum semiovale (upper images). Images at 3 months after intraarterial bevacizumab therapy reveal a marked decrease in FLAIR signal volume of $74 \%$ (lower images). Right: Brain T1-weighted contrast-enhanced MR images before treatment (upper images). There is a $34 \%$ decrease in contrast enhancement 3 months after intraarterial bevacizumab infusion (lower images).

\section{Role of VEGF in RN}

Vascular endothelial growth factor is a tyrosine kinase that plays an important role in angiogenesis and modulation of vascular permeability. VEGF-A binds with high specificity to VEGF receptor-1 and VEGF receptor-2 on vascular endothelial cells. These modulate downstream signaling pathways affecting various cellular processes. VEGF has recently been implicated in the pathophysiology of RN. Reactive astrocytes immediately surrounding the necrotic core in RN are strongly positive for VEGF by immunohistochemistry. ${ }^{19}$ It has been postulated that radiation causes microvascular injury leading to hypoxia. ${ }^{19}$ Hypoxia-induced VEGF upregulation then drives an increase in vascular permeability, leading to the extensive vasogenic edema seen in $\mathrm{RN}$.

\section{Bevacizumab Therapy for RN}

Bevacizumab (Avastin, Genentech BioOncology) is a recombinant humanized version of a murine anti-human VEGF monoclonal antibody. It binds circulating VEGF receptors with high specificity, blocking the downstream signaling cascade. Bevacizumab was originally developed and tested as an antiangiogenic treatment for various solid tumors.

More recently, intravenously administered bevacizumab was shown in a blinded, placebo-controlled, randomized trial $(n=14)$ to be effective in the treatment of refractory RN after radiation therapy in brain tumors. ${ }^{14}$ Patients received $7.5 \mathrm{mg} / \mathrm{kg}$ bevacizumab intravenously every 3 weeks for 4 cycles. All patients receiving bevacizumab, and none of the patients receiving placebo, had significant clinical and radiographic improvement. This improvement was durable, persisting at 10 months in 9 (92\%) of 11 patients. There was, however, a high rate of adverse events $(55 \%)$, and major adverse events occurred in $27 \%$. There have since been a handful of AVM RN cases in adults successfully treated with intravenous bevacizumab., ${ }^{7,20}$

There are significant known side effects of bevacizumab including gastrointestinal tract perforation, deep venous thrombosis, venous sinus thrombosis, pulmonary embolus, intracranial hemorrhage, wound dehiscence, and severe hypertension. ${ }^{1,6,11,12,21-23}$ These complications are common in the antiangiogenic class of drugs and reflect systemic exposure to bevacizumab. Current intravenous bevacizumab regimens use a dose of $7.5 \mathrm{mg} / \mathrm{kg}$ every 3 weeks for 4 cycles. Using a lower bevacizumab dose may lower systemic exposure and decrease complication rates, providing a rationale for further study. To maintain therapeutic efficacy of the drug at the lower dose, however, its ability to penetrate the brain must be improved. There are two ways to increase drug penetration into the brain: intraarterial route of administration and blood-brain barrier disruption.

\section{Intraarterial Delivery}

Intraarterial therapy decreases volume dilution of the drug in the circulation and reduces first-pass degradation through proteolytic catabolism. ${ }^{10}$ Superselective intraarterial injection of ${ }^{99 \mathrm{~m}} \mathrm{Tc}-$-hexamethylpropyleneamine oxime (Ceretec) into human cerebral arteries achieves a concentration of radiotracer in brain tissue 50 times higher than with intravenous injection. ${ }^{16}$ In clinical studies of cerebral chemotherapy, the concentration delivered to the tumor via intraarterial injection versus intravenous injection of chemotherapeutic agents was 5 times higher with hydrosoluble drugs and up to 50 times higher with liposoluble drugs. Intraarterial therapy does, however, involve an invasive angiographic procedure. Risk of significant procedure-related morbidity is less than $1 \%$, but can include stroke, allergic reaction to contrast dye, kidney impairment, and bleeding from the access site.

\section{Blood-Brain Barrier Disruption}

The BBB is a selective permeability barrier that blocks entry of many drugs into the brain. Bevacizumab is a monoclonal antibody with a high molecular weight (149 $\mathrm{kD}$ ). There is convincing evidence in the literature that the concentration in the brain of high-molecular weight molecules can be significantly increased after osmotic BBBD. ${ }^{2,18,27}$ Several tumor clinical trials have shown that localization of monoclonal antibodies to the brain is poor 
without BBBD $(0.0006 \%-0.0043 \%$ of the injected dose/ gram of tumor). ${ }^{4,8,13,17,25,26}$ There is also evidence of a 20 fold increase in permeability to immunoreactive immunoglobulin $\mathrm{M}$ monoclonal antibody with BBBD in rats. ${ }^{17}$ Based on these findings, BBBD may also significantly increase delivery of bevacizumab to the brain.

\section{Intraarterial Bevacizumab Infusion After Osmotic BBBD for Treatment of RN}

We used a combination of an intraarterial route of drug delivery and BBBD to reduce the bevacizumab dose while maintaining efficacy. This is supported by the durable clinical and radiographic response in our 2 patients after a single $2.5-\mathrm{mg} / \mathrm{kg}$ dose of bevacizumab. This approach may reduce the incidence of serious systemic toxicities compared with the intravenous bevacizumab regimen (7.5 $\mathrm{mg} / \mathrm{kg}$ every 3 weeks for 4 cycles).

The safety of intraarterial bevacizumab treatment after hyperosmotic BBBD was recently established in a small series of patients with malignant glioma. ${ }^{3}$ This was done through superselective injection of intracranial tumor arterial pedicles for the purpose of antitumor effects. Dose escalation was performed from $2 \mathrm{mg} / \mathrm{kg}$ to $15 \mathrm{mg} / \mathrm{kg}$ without reaching the maximal tolerated dose. There was a significant decrease in the contrast-enhancing and FLAIR signal characteristics of the tumor and surrounding brain at 1 month after treatment. Overall toxicity in this cohort was comparable to that in previous reports for intravenous bevacizumab therapy. In addition, hyperosmotic BBB breakdown followed by intraarterial bevacizumab administration did not cause any direct neurotoxicity; there were no cases of intracranial hemorrhage. In a different study, the same authors also performed extensive cost analysis of bevacizumab therapy for recurrent glioblastoma. ${ }^{5}$ They showed a $58 \%$ total cost savings for bevacizumab treatment when it was administered intraarterially as compared with intravenously. This savings was achieved despite the fact that the authors used a $15-\mathrm{mg} / \mathrm{kg}$ intraarterial dose of bevacizumab, which is 6 times higher than the concentration used in the present case series.

Herein, we have described 2 pediatric patients with highly symptomatic RN in the brain after SRS for the treatment of cerebral AVMs. The RN was refractory to all accepted medical therapies. Both patients were steroid dependent for a prolonged period and had developed severe cushingoid features. Both had suffered a significant decline in quality of life, suffering severe headaches and needing to withdraw from school. In both instances, the patients made a remarkable clinical and radiographic improvement after receiving a single low dose of intraarterial bevacizumab following hyperosmotic BBB breakdown. To our knowledge, this report marks the first demonstration of the successful treatment of cerebral RN using targeted intraarterial bevacizumab therapy.

\section{Conclusions}

We propose earlier consideration of bevacizumab treatment to avoid neurological morbidity and severe adverse effects of long-term steroid treatment. We have shown that intraarterial bevacizumab after hyperosmotic BBB break- down appears to be safe, effective, and durable treatment for medically refractory $\mathrm{RN}$ in the brain. Advantages of the intraarterial route over the intravenous route may include higher concentration of drug delivery to the targeted brain tissue, decreased systemic toxicity because of the much smaller dose of bevacizumab used, and the significantly lower cost. The main limitation of the present report is the very small number of patients. Future prospective clinical trials comparing intravenous and intraarterial bevacizum$\mathrm{ab}$ treatment in this setting deserve consideration.

\section{References}

1. Abbrederis K, Kremer M, Schuhmacher C: [Ischemic anastomotic bowel perforation during treatment with bevacizumab 10 months after surgery.] Chirurg 79:351-355, 2008 (Ger)

2. Armstrong BK, Robinson PJ, Rapoport SI: Size-dependent blood-brain barrier opening demonstrated with [14C]sucrose and a 200,000-Da [3H]dextran. Exp Neurol 97:686-696, 1987

3. Boockvar JA, Tsiouris AJ, Hofstetter CP, Kovanlikaya I, Fralin S, Kesavabhotla K, et al: Safety and maximum tolerated dose of superselective intraarterial cerebral infusion of bevacizumab after osmotic blood-brain barrier disruption for recurrent malignant glioma. Clinical article. J Neurosurg 114:624-632, 2011

4. Bullard DE, Bigner DD: Applications of monoclonal antibodies in the diagnosis and treatment of primary brain tumors. J Neurosurg 63:2-16, 1985

5. Burkhardt JK, Shin BJ, Schlaff CD, Riina H, Boockvar JA: Cost analysis of intra-arterial versus intra-venous delivery of bevacizumab for the treatment of recurrent glioblastoma multiforme. J Exp Ther Oncol 9:183-186, 2011

6. Chamberlain MC, Johnston S: Salvage chemotherapy with bevacizumab for recurrent alkylator-refractory anaplastic astrocytoma. J Neurooncol 91:359-367, 2009

7. Deibert CP, Ahluwalia MS, Sheehan JP, Link MJ, Hasegawa $\mathrm{T}$, Yomo S, et al: Bevacizumab for refractory adverse radiation effects after stereotactic radiosurgery. $\mathbf{J}$ Neurooncol 115:217-223, 2013

8. Epenetos AA, Courtenay-Luck N, Pickering D, Hooker G, Durbin H, Lavender JP, et al: Antibody guided irradiation of brain glioma by arterial infusion of radioactive monoclonal antibody against epidermal growth factor receptor and blood group A antigen. Br Med J (Clin Res Ed) 290:1463-1466, 1985

9. Flickinger JC, Kondziolka D, Lunsford LD, Kassam A, Phuong LK, Liscak R, et al: Development of a model to predict permanent symptomatic postradiosurgery injury for arteriovenous malformation patients. Int J Radiat Oncol Biol Phys 46:1143-1148, 2000

10. Gobin YP, Cloughesy TF, Chow KL, Duckwiler GR, Sayre JW, Milanese K, et al: Intraarterial chemotherapy for brain tumors by using a spatial dose fractionation algorithm and pulsatile delivery. Radiology 218:724-732, 2001

11. Grivas AA, Trafalis DT, Athanassiou AE: Implication of bevacizumab in fatal arterial thromboembolic incidents. J BUON 14:115-117, 2009

12. Heinzerling JH, Huerta S: Bowel perforation from bevacizumab for the treatment of metastatic colon cancer: incidence, etiology, and management. Curr Surg 63:334-337, 2006

13. Lee YS, Bullard DE, Zalutsky MR, Coleman RE, Wikstrand CJ, Friedman HS, et al: Therapeutic efficacy of antiglioma mesenchymal extracellular matrix 131I-radiolabeled murine monoclonal antibody in a human glioma xenograft model. Cancer Res 48:559-566, 1988

14. Levin VA, Bidaut L, Hou P, Kumar AJ, Wefel JS, Bekele BN, 
et al: Randomized double-blind placebo-controlled trial of bevacizumab therapy for radiation necrosis of the central nervous system. Int J Radiat Oncol Biol Phys 79:1487-1495, 2011 (Erratum in Int J Radiat Oncol Biol Phys 84:6, 2012)

15. Monaco EA III, Niranjan A, Kano H, Flickinger JC, Kondziolka D, Lunsford LD: Management of adverse radiation effects after radiosurgery for arteriovenous malformations. Prog Neurol Surg 27:107-118, 2013

16. Namba H, Kobayashi S, Iwadate Y, Saegusa T, Sato A, Watanabe Y, et al: Assessment of the brain areas perfused by superselective intra-arterial chemotherapy using single photon emission computed tomography with technetium-99m-hexamethyl-propyleneamine oxime-technical note. Neurol Med Chir (Tokyo) 34:832-835, 1994

17. Neuwelt EA, Barnett PA, Hellström KE, Hellström I, McCormick CI, Ramsey FL: Effect of blood-brain barrier disruption on intact and fragmented monoclonal antibody localization in intracerebral lung carcinoma xenografts. J Nucl Med 35:1831-1841, 1994

18. Neuwelt EA, Frenkel EP, D’Agostino AN, Carney DN, Minna JD, Barnett PA, et al: Growth of human lung tumor in the brain of the nude rat as a model to evaluate antitumor agent delivery across the blood-brain barrier. Cancer Res 45:2827-2833, 1985

19. Nonoguchi N, Miyatake S, Fukumoto M, Furuse M, Hiramatsu R, Kawabata S, et al: The distribution of vascular endothelial growth factor-producing cells in clinical radiation necrosis of the brain: pathological consideration of their potential roles. J Neurooncol 105:423-431, 2011

20. Sadraei NH, Dahiya S, Chao ST, Murphy ES, Osei-Boateng $\mathrm{K}, \mathrm{Xie} \mathrm{H}$, et al: Treatment of cerebral radiation necrosis with bevacizumab: The Cleveland Clinic experience. Am J Clin Oncol [epub ahead of print], 2013

21. Suenaga M, Mizunuma N, Kobayashi K, Shinozaki E, Matsusaka S, Chin K, et al: Management of venous thromboembolism in colorectal cancer patients treated with bevacizumab. Med Oncol 27:807-814, 2010

22. Takano M, Kikuchi Y, Kato M, Yoshikawa T, Kita T: [Bowel perforation associated with bevacizumab therapy in recurren ovarian cancers without bowel obstruction or bowel involvement.] Gan To Kagaku Ryoho 35:1981-1984, 2008 (Jpn)
23. Vredenburgh JJ, Desjardins A, Kirkpatrick JP, Reardon DA, Peters KB, Herndon JE II, et al: Addition of bevacizumab to standard radiation therapy and daily temozolomide is associated with minimal toxicity in newly diagnosed glioblastoma multiforme. Int J Radiat Oncol Biol Phys 82:58-66, 2012

24. Williamson R, Kondziolka D, Kanaan H, Lunsford LD, Flickinger JC: Adverse radiation effects after radiosurgery may benefit from oral vitamin $\mathrm{E}$ and pentoxifylline therapy: a pilot study. Stereotact Funct Neurosurg 86:359-366, 2008

25. Zalutsky MR, Moseley RP, Benjamin JC, Colapinto EV, Fuller GN, Coakham HP, et al: Monoclonal antibody and $\mathrm{F}(\mathrm{ab}) 2$ fragment delivery to tumor in patients with glioma: comparison of intracarotid and intravenous administration. Cancer Res 50:4105-4110, 1990

26. Zalutsky MR, Moseley RP, Coakham HB, Coleman RE, Bigner DD: Pharmacokinetics and tumor localization of 131Ilabeled anti-tenascin monoclonal antibody 81C6 in patients with gliomas and other intracranial malignancies. Cancer Res 49:2807-2813, 1989

27. Ziylan YZ, Robinson PJ, Rapoport SI: Differential bloodbrain barrier permeabilities to $[14 \mathrm{C}]$ sucrose and $[3 \mathrm{H}]$ inulin after osmotic opening in the rat. Exp Neurol 79:845-857, 1983

\section{Author Contributions}

Conception and design: Dashti, LaRocca. Acquisition of data: Dashti, Yao. Analysis and interpretation of data: Dashti, Kadner, LaRocca. Critically revising the article: Spalding, Sun, LaRocca. Reviewed submitted version of manuscript: all authors. Approved the final version of the manuscript on behalf of all authors: Dashti. Administrative/technical/material support: Kadner, Kumar. Study supervision: LaRocca.

\section{Correspondence}

Shervin R. Dashti, 4950 Norton Healthcare Blvd., Ste. 205, Louisville, KY 40241. email: shervin.dashti@nortonhealthcare. org. 